\title{
Hearing loss and cognition: a discussion for audiologists and hearing healthcare professionals
}

\begin{abstract}
and goals
Among researchers, clinicians and patients, there is widespread and growing interest in the relationship between hearing and cognition. The Cognition in Hearing Special Interest Group (SIG) is part of the British Society of Audiology (BSA) and is uniquely positioned to explore the relationship between hearing loss, amplification and cognitive ability and cognitive decline. The multiplicity of emerging reports concerning hearing loss and cognition is increasing rapidly. In light of this vast growth, there is a risk that clinicians may be left uncertain regarding the nature and extent of the emerging evidence linking hearing and cognition. The trickle-down corollary of such uncertainty can negatively impact patient care. Answering challenging questions and disseminating complex information about the latest evidence-based hearing science are a daily part of any clinician's role and those in audiology services may be asked "How does my hearing loss affect my chance of getting dementia?" or "can hearing aids help people with dementia?" This discussion is therefore, based on articles and information our committee members selected to represent the status quo.

The Cognition in Hearing SIG aims, through this discussion article, to provide clinicians a contemporary understanding of research on this topic. We will discuss evidence concerning hearing loss and cognition and how it relates to people living with hearing loss and cognitive decline or dementia, and we shall pose some challenges and opportunities for future research and clinical practice evidence. Therefore, to address these aims in an accessible manner for clinicians, the Cognition in Hearing SIG shall address the following broad questions:
\end{abstract}

a) What is the relationship between hearing loss and cognition?

b) What do we know about hearing loss and cognitive performance?

c) Is there a link between hearing loss, cognitive decline, and dementia?

d) Can we intervene on the relationship between hearing loss and cognition?

Keywords: audiology, hearing loss, hearing aids, dementia, cognition
Volume 12 Issue 3 - 2020

\author{
Douglas L Beck,' Sarah Bant, ${ }^{2}$ Nathan A \\ Clarke $^{3}$ \\ 'Vice President of Academic Sciences, Oticon Inc., Adjunct \\ Professor of Communication Disorders and Sciences, State \\ University of New York at Buffalo, USA \\ ${ }^{2}$ Principal Clinical Scientist, Betsi Cadwaladr University Health \\ Board, UK \\ ${ }^{3}$ Candidate \& Clinical Audiologist, National Institute for Health \\ Research (NIHR) Nottingham Biomedical Research Centre, UK
}

Correspondence: Douglas L Beck, Vice President of Academic Sciences, Adjunct Professor of Communication Disorders and Sciences, State University of New York at Buffalo \& Vice Chair of the Cognition in Hearing SIG, USA,

Email douglaslbeck@gmail.com

Received: April 16, 2020 | Published: May 14, 2020
Abbreviations: SIG, special interest group; BSA, british society of audiology; MMMSE, modified mini-mental state examination; DSS, digit symbol substitution

\section{Overview of hearing loss and cognition research}

Hearing loss causes communication difficulty as a primary effect and negatively impacts the hearing-related quality of life in older adults. Sensory and cognitive function decrease as age increases, and sensory impairments (including age-related hearing loss) are well-evidenced to be risk factors for age-related cognitive decline and dementia. One of the earlier studies to show this was Lin et al., ${ }^{1}$ who studied 1984 older adults. Their baseline cohort consisted of participants without cognitive impairment as measured on the Modified Mini-Mental State Examination (MMMSE) all of whom underwent audiometric testing and were followed for 6 years. In total, 1162 individuals with baseline hearing loss (pure-tone average $>25$ dB) had annual rates of decline in the MMMSE and Digit Symbol Substitution (DSS) test scores that were $41 \%$ and $32 \%$ worse than those for individuals with normal hearing. Compared to those with normal hearing, individuals with hearing loss had a $24 \%$ increased risk for incident cognitive impairment. The rates of cognitive decline and the risk for cognitive impairment were found to be linearly associated with the severity of the hearing loss. The authors concluded that hearing loss is independently associated with accelerated cognitive decline and incident cognitive impairment in community-dwelling older adults. This has been supported since by numerous studies, including from researchers across the globe, and now in combined meta-analyses.

\section{Types of evidence}

Epidemiological studies offer the opportunity to discover and reveal the frequency and pattern, and causes and risk-factors, of health-related states and events. Epidemiological studies gather selfreports or test results from large numbers of participants in an attempt to account for the many ways in which people vary. Resultantly, we can see trends and understand the scale on which multiple conditions occur together, or the variation when an intervention is used, but it is not possible to determine cause and effect. Key examples can be found among the first studies to raise the profile of dementia and hearing loss in such a significant way. ${ }^{2,3}$ Epidemiological studies may be conducted over many years to quantify decline in abilities or 
conditions $^{1}$ or the difference between groups of people ${ }^{4,5}$ or in a group, pre-and-post intervention. ${ }^{6}$ Changes over time can also be studied with observational and experimental studies. These tend to be more specific to the condition, such as observing cognitive changes over time in those with hearing loss, and may use multiple groups at the same time, such as people who choose to wear hearing aids, versus people who choose not to wear hearing aids. Again, "cause" cannot directly be inferred. However, it is well-evidenced that people who choose to use hearing aids tend to have greater self-efficacy and social interaction even before they take up hearing aids. ${ }^{7}$ Alternatively, one can account for this by studying people experimentally before and after a change is made. ${ }^{8,9}$

While attempting to gain conclusive answers to questions, researchers ideally turn to conducting randomized controlled trials (RCT). For example, RCTs may consider the benefits of hearing aid use for cognitive performance, in which participants are assigned blindly to either treatment or placebo groups. It is possible to set a hearing aid to a 'placebo setting'. ${ }^{10}$ However, such studies encounter problems in other ways. Firstly, the ethics regarding denying working hearing aids to willing participants for long enough to study the effect of that denial; the study that used placebo devices for this purpose limited the delay in true hearing aid use to only 6 months for the control group. ${ }^{10}$ Secondly, if focusing on those that are not interested in hearing aids, while ethically palatable, there tends to be poor compliance with the study conditions, i.e. participants effectively using the hearing aids in their daily life. ${ }^{11}$ Ironically, it is this latter group of individuals that are possibly the most interesting, being the least studied in terms of cognitive decline, and those who might benefit most from healthy aging campaigns. Current RCT studies tend to compare hearing aid use within differing models of rehabilitation in order to increase understanding in a more ethical manner. ${ }^{12,13}$

In the case of complex disorders and complex interventions, such as hearing loss and amplification, we may turn to qualitative research. This is an approach in which we listen to the voice of the person, i.e. people living with hearing loss and/or with dementia. By drawing from the experiences of people living with a disorder and/ or using an intervention, we begin to understand the complexity of the landscape from different viewpoints, and either combine this with emerging quantitative evidence to better evidence hypotheses, or use the experiential findings as a starting point for further enquiry. These approaches have been used to understand the experiences of people who are deaf and have dementia ${ }^{14}$ and people living with sensory impairment and dementia. ${ }^{15}$

\section{Measuring hearing}

In addition to challenges from differing research methodologies, we are also limited by what we choose to measure. Typically, hearing is quantified based on pure tone thresholds. Nonetheless, we are aware that the functional integration, capacity or outcome of the auditory system cannot be derived based solely on pure tone thresholds. That is, audiograms only reflect pure tone thresholds across a limited spectrum $(250-8000 \mathrm{~Hz})$. Notably, these same pure tones are rarely, if ever, heard in the real world. Further, the end-game for humans is not to simply hear/perceive artificial sounds, but is, to comprehend and apply meaning (primarily) to speech sounds; to listen. Indeed, "Listening is where hearing meets brain". ${ }^{16}$ Listening is much more than the threshold at which one perceives a pure tone. Listening occurs within the brain and listening is the "end-game." Listening is clearly built on a foundation of hearing, yet listening involves cognition, attention, intention, vocabulary, processing ability, processing speed, working memory, short term and long-term memory and more, and occurs within the brain itself.

Although age-related hearing loss is typically characterised by loss of outer hair cells in the cochlea, which are reflected via the audiogram, arguably, the more important auditory phenomena happens after the sound (bio-electric energy) exits the cochlea and synapses with auditory nerve fibres and the resultant bio-electric signal is sent to both superior temporal lobes of the brain, along the central auditory pathway. The stimuli may have substantial emotional impact which may involve the frontal lobes, and there may be redundant visual cues, thereby involving the occipital lobe and more for processing and interpretation. That is, for simple stimuli such as a pure tone, one might argue the ability to perceive the stimuli is enough. However, for language-based listening tasks, and to understand and apply meaning to speech sounds, interactions within and throughout the brain are vast, likely innumerable, and it is the brain's interpretation of sound (from the ear) from which we derive meaning.

Further, it is well-known that people with similar (or identical) hearing thresholds often have incredibly different abilities to comprehend, understand or untangle sound into meaningful percepts. Further, speech recognition in quiet (SIQ) does not necessarily correlate with speech recognition in noise (SIN) and there is no constant or predictable relationship between SIQ and SIN. Of note, although some 38 million people (USA) have hearing loss as demonstrated on an audiogram, there are an additional 26 million people $^{17}$ who have hearing difficulty and/or SIN problems which cause a multitude of problems with regard to small and large group communication, depression, anxiety, stress, social isolation, quality of life, and more. There are many possible reasons for hearing difficulties without the presence of peripheral hearing or specific central auditory hearing disorders, one of which is cognitive ability.

\section{Measuring cognition}

A similar situation is found with cognitive ability: despite the ability to measure some "relatively specific" aspects (i.e., the "greatest hits") of human cognition, information processing abilities such as short and long-term and working memory, learning ability, attention, pattern recognition and more, ${ }^{18}$ we are at a loss to $100 \%$ thoroughly or entirely accurately define things like human consciousness and human cognitive ability. This poses an additional challenge when specifically considering cognitive ability related to hearing, or the interaction between cognitive decline and hearing loss. Boogert, Madden and colleagues ${ }^{19}$ stated "...cognitive abilities cannot be directly observed, they must be inferred..." The authors state all animals learn, remember and integrate sensory provided information to achieve decisions and determine behaviours. However, how, why and when these cognitive abilities evolve and mature is a mystery, with significant inter and intra-individual variation. In essence, whichever cognitive attribute we measure in an individual at a given moment in time, likely does not represent their entire cognitive ability, and importantly, whatever the outcome of that measured attribute happens to be, it can change over time. That is; the measurement of sub-skills only measures the particular sub-skill at a given moment in time. Sub-skills such as perception, memory, math or verbal skills are important, are very useful to compare the ability of individuals within that same domain. However, the ability to predict overall cognitive ability based on one or multiple sub-skills is not a proven attribute. 
Rowe and Healy ${ }^{18}$ reported that variability in a cognitive task does not necessarily demonstrate individual variation in cognitive ability. They report that demonstrating more memory, faster learning or finer discriminations may not always be better - and indeed, they cautioned we need to design more stringent tests and we must be more cautious regarding interpreting results. Furthermore, recent work suggests that the types of cognitive test may be important for successfully understanding associations between complex constructs such as hearing and cognition. For example, in a recent systematic review and meta-analyses of associations between tinnitus and cognitive performance, Clarke et al., ${ }^{20}$ found that complex tasks requiring cognitive control and executive functioning demonstrated reliable associations with tinnitus. Given previous equivocal and mixed results frequently reported within the literature, this analysis demonstrates that cognition is not a monolithic construct and it is crucial to give due consideration to which elements/sub-skills are being measured. With an understanding of the types of evidence available and the various ways that cognition and hearing loss can be measured, we can return to the discussion questions posed earlier. In order to present the current evidence available, the Cognition and Hearing SIG has selected evidence-based discussions drawn from topics of interest expressed during various clinical encounters, and have summarized the latest understanding on these, along with examples of recent research relating to these topics.

\section{Discussion questions}

\section{What is the link between hearing loss and cognition?}

Even among experts, the word 'cognition' is understood in a variety of different ways depending on the context in which it is used; some experts use the term to refer to the contents of thoughts, while others use it to refer to the efficiency of thought processes. ${ }^{21}$ Further, it is important to distinguish between cognitive performance and cognitive decline. Decrements in cognitive performance through auditory deprivation can be subtle, and are experienced by most individuals. Cognitive decline encompasses mild cognitive impairment (MCI) and dementias and neuro-cognitive disorders, which occur when cognitive performance is functionally impaired as indicated and measured through screening protocols and questionnaires and diagnostic tests (respectively), and is typically corroborated with medical imaging.

When we discuss the link between hearing and cognition, we're typically addressing the difference between 'hearing' with the ears, and 'listening' with the brain (see above). As such, fundamentally, the function of the ears is to transduce a signal to the brain, which subsequently processes it and assigns meaning to it, through the act of cognition. Another context we are typically interested in is the relationship between hearing loss and cognitive performance. However, cognition is known as a latent variable and cannot be directly observed. Cognition is typically measured using established cognitive and neuropsychological tests. Regarding hearing loss and cognitive decline, epidemiological and longitudinal studies exist that have followed and assessed patients over the course of their lives with reports of an accelerated cognitive decline with advancing age in older adults with hearing loss when compared to those with no hearing loss. Recently, Amieva and Ouvard ${ }^{22}$ reported that despite multiple caveats (the scarce number of studies, contrasting results, the absence of interventional studies such as random controlled studies) "the available data globally support the hypothesis that hearing aids have a positive impact on long-term cognition in older adults suffering from hearing loss." Glick and Sharma ${ }^{7}$ reported Age Related Hearing Loss (ARHL) is associated with cognitive decline and functional and structural brain changes. They determined that multiple deficits were improved after 6 months of daily hearing aid use, providing striking evidence of compensatory cortical neuroplasticity. In particular they noted a reversal in cross-modal reorganization, as well as gains in speech perception and cognitive performance.

Further, various scientific links and hypotheses have been proposed between hearing loss and cognition to explain how hearing loss may link to progressive cognitive decline and also dementia:

1. Common causes: Both hearing loss and cognitive decline sharing the same widespread neurodegenerative causes.

2. Cascade via social effects: The experience of hearing loss cascading into social disengagement, loneliness and depression, cascading to accelerated brain atrophy and accelerated cognitive decline and dementia.

3. Cascade via auditory deprivation: Auditory deprivation resulting in impoverished cortical input, causing neuroplastic changes, cascading into cognitive decline and dementia.

4. Cognitive load: Hearing loss causing cognitive resources to be diverted from memory function into auditory processing, adding to the cognitive load, and leading to cognitive decline and dementia.

Of note, hypotheses 2, 3 and 4 (above) are of causal effects, whereas the first is co-occurring and not due to auditory deprivation from hearing loss per se. Livingston et al., ${ }^{23}$ explain that older age factors and microvascular pathology increase the risk of both dementia and peripheral hearing loss and the presence of this might, therefore, confound any causal associations. Indeed, hearing loss has been described as a marker for poor health; those with unhealthy lifestyles (smoking, alcohol, poor diet, lack of exercise) being at greater risk of hearing loss and of cognitive decline/dementia. ${ }^{24}$ It is reasonably clear from these two hypotheses that "hearing impairment is not good for the brain" 25 and rather than one single hypothesis being correct, the true picture may be a combination of multiple hypothesis. Uchida et al., ${ }^{26}$ concluded it is likely that multiple mechanisms occur in differing extents for each person.

\section{What effect does hearing loss have on cognitive performance?}

Difficulty understanding speech in the presence of background noise is the most common complaint among those with hearing loss and listening difficulty. Struggling to perceive speech in the presence of competing sound, may be due to a peripheral hearing loss that can be supported with hearing aids, or indeed a disorder of the central auditory pathway; however, it may instead or additionally be a cognitive issue. As mentioned previously, although effects may be subtle, laboratory studies have effectively demonstrated that auditory deprivation can impair cognitive performance. For instance, seminal studies have shown that even for normal hearing individuals, a reduction in the signal-to-noise ratio can reduce a person's memory for spoken words. ${ }^{27,28}$ These remarkable findings are particularly true for people with hearing loss, with similar experiments showing that adults with hearing loss perform more poorly compared to those without. ${ }^{29,30}$ 
When considering the effect that hearing loss has on cognitive performance, we may consider which areas of cognition may display poorer performance in challenging, noisy conditions. Dryden et al., ${ }^{31}$ completed a meta-analysis of 25 studies assessing associations between cognitive performance and speech-in-noise (SIN) perception. They reported overall association between cognitive performance and SIN perception was a moderate positive correlational relationship $(\mathrm{r}=.31)$ For component cognitive domains, the association with (pooled) SIN perception was as follows: processing speed $(\mathrm{r}=.39)$, inhibitory control $(\mathrm{r}=.34)$, working memory $(\mathrm{r}=.28)$, episodic memory $(\mathrm{r}=.26)$, and crystallized IQ $(\mathrm{r}=.18)$. Similar associations were shown for the different speech target and masker types. However, a crucial and more familiar aspect of this relationship for clinicians is the nature of the hearing loss that is being considered. For example, presbycusis is the most prevalent form of hearing loss and Rogers and Peele ${ }^{32}$ note that a resilience to age-related decline in some memory systems may play an important role in speech perception and subsequent agerelated cognitive performance in acoustically adverse and challenging conditions. Further, the issues at hand may not be limited by traditional categories/degrees of hearing and hearing loss. In some populations, it appears that even for people within "normal hearing categories," as hearing ability decreases along the continuum from "excellent" hearing to "normal" hearing, specific measurable aspects of cognition decrease.$^{33}$ That is, among people classically considered to have normal hearing, as their hearing ability decreases, so too, does cognition, in a clinically meaningful way.

However, evidence can be mixed when taken out of the laboratory. For example, Mamo, Reed, Sharrett et al., ${ }^{34}$ reported on older adults (mean age 78 years) in whom poorer cognitive performance (with specific regard to memory, language, executive functioning and global function) was associated with worse speech-in-noise performance, regardless of hearing thresholds and demographic and disease factors. The authors suggest the possibility that people with more significant hearing loss than those in the study would be more impacted by their cognitive processing abilities given their greater hearing challenges of understanding speech-in-noise. While Gaeta, Azzarello, Baldwin et al. ${ }^{35}$ examined the effect of audibility on the Mini-Mental State Examination (MMSE) and concluded reduced audibility does indeed, have a negative effect on MMSE scores in "cognitively intact" participants.

\section{Is there a link between hearing loss, cognitive decline, and dementia?}

Perhaps the most pressing concern for patients with hearing loss is understanding whether having hearing loss will increase their chance of cognitive decline and ultimately lead to dementia. Deal, Betz, Yaffe et al. ${ }^{36}$ reported a $10 \mathrm{~dB}$ increase in hearing thresholds may indicate a clinically meaningful $14 \%$ increased risk for dementia. They concluded that hearing loss is associated with an increased risk of developing dementia in older adults. Livingston, Sommerland, Ortega et al. ${ }^{23}$ reviewed that evidence base at the time and were unable to find systematic reviews on hearing loss and dementia. The authors pooled the results from three relevant primary studies, and noted that hearing loss was a significant risk factor for dementia. Livingston and colleagues suggested that approximately two thirds of dementia risk is genetic and one third of dementia risk is likely attributable to a combination of nine risk factors; education to a maximum of age 11-12 years, midlife hypertension, midlife obesity, hearing loss, late life depression, diabetes, physical inactivity, smoking, and social isolation. Among the 9, hearing loss was found to be the most significant factor, with a Population Attributable Factor (PAF) of $9 \%$. They stated that the mechanism underlying cognitive decline associated with peripheral hearing loss was not yet clear; nor was it established from the evidence whether 'correction' of hearing, such as with hearing aids, can prevent or delay the onset of dementia. This paper was welcomed by audiology clinicians for its synthesis of the evidence on the risk resulting from hearing loss, placed in the context of the risks from other disorders, which helps highlight hearing loss as a key modifiable disorder, i.e. one that individuals concerned about dementia can take action to change. At the same time, researchers cautioned against leaping to the conclusion that adopting hearing aids reduces the risk by this amount. ${ }^{37}$

Yuan, Sun, Sang, Pham and Kong ${ }^{38}$ published a meta-analysis of 11 cohort studies to assess published evidence for an association between peripheral hearing function and central hearing function with cognitive impairment in older adults (defined as a cohort mean age of 60 years old or greater at baseline). Pooled results indicated the risk of cognitive impairment for those with moderate to severe hearing impairment (average PTA $>40 \mathrm{~dB}$ HL) was $29-57 \%$ greater than those with normal hearing. For all participants with average hearing thresholds of $>25 \mathrm{~dB}$ HL, the risk of cognitive impairment was $29 \%$ greater compared to those with no hearing impairment. A doseresponse trend was found between peripheral hearing function and cognition, whereby for every $10 \mathrm{~dB}$ increase in hearing loss, there was an estimated $12 \%$ increase in the incidence of cognitive impairment. The authors reported their findings support the contribution of peripheral and central hearing dysfunction to the risk of cognitive impairment in the older population. Further, the association between central hearing dysfunction and cognitive impairment was stronger than the association between peripheral hearing dysfunction and cognitive impairment.

Jafari, Kolb and Mohajerani ${ }^{39}$ reviewed the literature and reported that age-related hearing loss is the third leading cause of chronic disability in older adults, and has been shown to be associated with predisposing cognitive impairment and dementia. The authors state the likelihood of significant hearing loss (average PTA $>25 \mathrm{~dB}$ HL) for people ages $60-80$ years is approximately $25 \%$, and for those aged 70 years and older, $65 \%$. They note a large body of evidence demonstrates age-related hearing loss is detrimental to physical and mental health, cognition, independence, social interaction, and quality of life in the elderly and hearing loss can precipitate early landmarks of dementia and Alzheimer's disease. As hearing loss increases, it manifests via profound consequences on verbal communication, and social, functional, and psychological well-being of the person. Hearing loss, depression, and anxiety are considered as the leading causes of disability worldwide, and can promote the occurrence of one another. The authors report hearing loss is associated with a multitude of health outcomes, similar to those identified for depression, anxiety, and stress, such as increased risk of cognitive impairment and dementia, reduced quality of life, low level of activity, frailty, social isolation, and poor general health. Dysfunction in social communication can be seen in individual's inability in coping with stressful situations and in managing personal, interpersonal, or geographic environments. Hearing loss, which is a source of stress and mental fatigue, can lead to social isolation and subsequent depression which may predispose individuals to cognitive decline.

With due consideration, the above findings represent multiple significant issues for clinicians; having to provide amplification and improve the quality of hearing for those who are already experiencing 
cognitive decline. It is well-known that a high percentage of those attending dementia assessment services have hearing loss. Allen et al., ${ }^{40}$ found $87 \%$ of those attending dementia assessment had hearing loss on measurement and $80 \%$ were unaware of this. Livingston et al., ${ }^{23}$ Uchida et al. ${ }^{26}$ Ray et al., ${ }^{41}$ and Panza et al., ${ }^{42}$ discuss the ways in which hearing loss may directly affect diagnosis and experience of dementia. This may be even more of an important clinical issue when cognitive assessments are instructed and/or performed verbally. This may also be a real experience of hearing loss exacerbating or mimicking symptoms of dementia, with many similarities shared such as in communication and language skills, ability to complete tasks of daily living independently, poor access to social and environmental cues, etc. Conversely, the same four papers also include that neurodegeneration with Alzheimer's Disease may result in increased hearing challenges in higher parts of the brain. Everyday effects of this may include reduced understanding of speech in noisy environments or with competing speakers, poorer concentration and attention, and a greater chance of overload from sounds. The authors suggest/ recommend that, as this presents in early stages, hearing assessments should include tests of peripheral and central function.

\section{Can we intervene on the relationship between hearing loss and cognition?}

Perhaps the most important question concerns intervention. Depending on the outlined scientific links between hearing loss and cognition, intervention could take various forms; from familiar hearing aids, to emerging interventions such as cognitive training, as well as established lifestyle interventions (e.g. reducing social isolation or improved nutrition). The different options for intervention are not mutually exclusive, and it is likely they can support one another to increase efficacy. Stahl ${ }^{25}$ reported interventions which delay the onset of dementia by merely one year would result in a greater than $10 \%$ decrease in the global prevalence of dementia in 2060. However, effective decision-making requires provision of evidence-based information on the effects of hearing loss, and also evidence-based information on the benefit of rehabilitation options.

Hearing aids are arguably the most familiar intervention for clinicians when it comes to potentially remediating hearing impairment and (potentially) cognitive decline. Modern hearing aids incorporate technological inventions in sound signal processing, direct streaming from the phone, convenient usage, and physical appearance. Improvements in wireless technology, frequency-specific amplification, background noise cancellation, directionality in conversation with multiple speakers, multi-speaker access technology, acoustic feedback cancellation and elimination, and decreased artifacts have led to impressive outcomes in speech perception in both quiet and noisy environments for adults.

Amieva et al., ${ }^{4}$ reported one of the first large-scale $(n=3670)$ prospective population-based studies assessing the association between self-reported hearing loss and cognitive trajectories over a 25-year period for three groups: those with that reported hearing loss but no hearing aid use ("unaddressed hearing loss"), those that reported hearing loss and hearing aid use, and those that reported no hearing loss. About $4 \%$ of participants reported severe hearing loss, $31 \%$ reported moderate hearing loss and the remaining $65 \%$ reported no hearing impairment. At follow-up, measures of global cognitive performance and cognitive complaints, functional ability, and symptoms of depression were acquired. Unaddressed self-reported hearing loss was associated with a faster rate of cognitive decline than was determined for hearing aid users and those that did not report hearing loss. The authors explained that this relation is potentially mediated by other factors, including social isolation and depressive symptoms, rather than a direct effect of hearing loss on cognitive decline. Moreover, when Amieva and colleagues provided additional control for depressive symptomology and other confounding factors, they did not identify a difference in cognitive decline for participants with or without self-reported hearing loss and with or without hearing aid use. They concluded that addressing one's hearing loss by use of hearing aids may slow cognitive decline by alleviating communication difficulties and improving mood and social interactions.

Deal et al., ${ }^{43}$ reported preliminary results of 40 adult participants in an RCT (aged 70-84 years) with untreated, adult-onset, bilateral, mild-to-moderate hearing loss who did not have dementia. Participants were randomized into either a "best-practices hearing" (BPH, i.e., rehabilitation with hearing aids) or "successful aging" (SA, rehabilitation without hearing aids) intervention. Both interventions were delivered over the course of four, 1-hour sessions every 1-3 weeks post-randomization. For the BPH intervention, participants engaged in the development of self-managed abilities for hearing loss and communication and were also offered assistive listening devices that could be used in conjunction with the hearing aids. The SA intervention consisted of an individually tailored interactive health education program for older adults based on the 10 Keys to Healthy Aging. ${ }^{44}$ In the $\mathrm{BPH}$ group, there was an improvement in the cognitive domain score for memory. In the SA group, results for proximal outcomes were consistent with no change or worse function. This study is ongoing, with results of the full-scale RCT expected from the ACHIEVE project team in 2022.

Jafari, Kolb and Mohajerani ${ }^{39}$ report advantages of hearing amplification devices in at least partially restoring hearing ability and improving overall cognitive performance in older adults. They report a successful aural rehabilitation program is a multi-stage approach that begins with counseling and identifying communication needs based on hearing and speech test results, interview and self-assessment questionnaires, communication situations, physical and mental health, and individual and family expectations. Ray, Popli and Fell ${ }^{5}$ assessed the link between memory as reflected in word recall, and executive function as reflected via verbal fluency, with hearing acuity derived from the HearCheck screener device. The eligible cohort from the English Longitudinal Study of Aging of people aged 50 years old and over consisted of 4,072 (55.1\%) women, with mean age of 67.4 years old (standard deviation 9.4 years). Of these, 3,056 (41.4\%) had mild hearing loss and $755(10.2 \%)$ had severe hearing loss. A total of 834 $(11.3 \%)$ used a hearing aid. The authors examined the association between hearing loss and cognition, while controlling for age, social isolation, sex, non-white race, whether the person lives alone, uses hearing aid(s), works, educational qualifications, is a current smoker, and has diabetes or hypertension. Across all individuals, there was a negative association shown between cognitive functioning and hearing loss. The authors concluded that untreated hearing loss is linked to a decline in cognitive function. Further, the odds of being socially isolated for those with mild hearing loss, compared to those with no hearing loss, increased as hearing loss increased which suggested that social isolation may act as a mediating factor.

As described earlier, one's cognitive ability on certain test measures, or even overall, is not the only consideration here. Hearing rehabilitation (amplification with hearing aids or other 
personal devices, alongside individualized behavior change support, counseling and communication training) has other known benefits to the individual and to society. Hearing loss fundamentally impacts one's ability to communicate and live one's daily life independently and within the context of society. Hearing aid use, supplied with rehabilitation described, is known to reduce the impact of this disability. Jilla et al., ${ }^{45}$ report that, despite the fact that only 1 in 5 people with hearing loss pursues amplification, for those who do, self-reported daily use of hearing aids was associated with increased satisfaction and an increased quality of life. Although hearing aids are most familiar, other promising interventions exist such as cognitive training, which may be useful in remediating detriments to cognitive performance in people with hearing loss, as well as improving aspects of speech in noise performance. This is a particularly attractive intervention option due to ease and cost-effectiveness associated with home-based, computerized training interventions. Lawrence et al., ${ }^{46}$ performed a systematic review and meta-analysis of nine studies which met their inclusion criteria, and these preliminary results suggest that home-based auditory training and cognitive training may improve cognition. More high-quality RCTs with long-term followup assessments are needed to determine whether auditory, cognitive, or a combined auditory-cognitive intervention is most efficacious for improving cognitive function in adults with hearing loss

Despite these emerging reports of benefit or delayed degradation of cognition with amplification, we are only at the beginning of our understanding of these relationships. That is, we realize much more information is required to make definitive causal statements. That is, in the early days of 2020, it appears increasingly clear that a universal and well-defined causation statement (such as " $\mathrm{X}$ causes $\mathrm{Y}$ ") is unlikely to be reported in the near-term future. The evidence that is emerging at present though, does show that for those that choose to identify and take action regarding their age-related hearing loss may also be impacting their age-associated cognitive trajectory and possibly reducing their incidence of dementia. From a public health messages perspective, or indeed within an audiology clinic, we can at least encourage those that are aware of their hearing loss and keen to take action. The message becomes clearer to the public and our patients if we speak about unmanaged hearing loss, rather than hearing loss. From a public health perspective, the consideration is two-fold: evidence and risk. The latest evidence has shown that hearing aids are an effective intervention with a negligible risk, ${ }^{47}$ while the risk of leaving hearing loss unmanaged could be costly in many areas: hearing outcomes, cognitive decline, and ultimately, patient quality of life.

\section{Summary}

Through identifying and answering questions that are pertinent to audiologists and hearing healthcare professionals, we have provided an evidence-based discussion of the current understanding regarding the complex relationship between hearing loss and cognition. This overview reflects BSA Cognition and Hearing SIG membership, knowledge, and interests, and fulfils a crucial role in keeping clinicians (and consequently) their patients up to date on the latest developments in this fast-evolving field. In doing so, we have described the observed links between hearing loss and cognition, and provided an overview of the various scientific theories that link them. We have discussed the known detrimental effects of hearing impairment on cognitive performance, and the various domains of cognition that have been implicated as having an important role in successful listening. We have also provided an overview of the relationship between hearing loss and cognitive decline, and discussed which interventions are currently available or being developed to support those affected by these issues, to remediate the known issues associated with hearing loss and cognition.

\section{Acknowledgments}

None.

\section{Conflicts of interest}

The author declares that there is no conflict of interest to disclose.

\section{Funding}

None.

\section{References}

1. Lin FR, Yaffe K, Xia J, et al. Health ABC Study Group. Hearing loss and cognitive decline in older adults. JAMA Intern Med. 2013;173(4):293-299.

2. Lin FR, Ferrucci L, Metter EJ, et al. Hearing loss and cognition in the Baltimore Longitudinal Study of Aging. Neuropsychology. 2011;25(6):763-770.

3. Lin FR, Metter EJ, O'Brien RJ, et al. Hearing loss and incident dementia Archives of Neurology. 2011;68(2):214-220.

4. Amieva H, Ouvrard C, Giulioli C, et al. Self-reported hearing loss, hearing aids, and cognitive decline in elderly adults: A 25-year study. Journal of the American Geriatrics Society. 2015;63(10):2099-2104.

5. Ray J, Popli G, Fell G. Association of Cognition and Age-Related Hearing Impairment in the English Longitudinal Study of Ageing. JAMA Otolaryngol Head Neck Surg. 2018;144(10):876-882.

6. Maharani A, Dawes P, Nazroo J, et al. Longitudinal relationship between hearing aid use and cognitive function in older Americans. Journal of the American Geriatrics Society. 2018;66(6):1130-1136.

7. Glick HA, Sharma A. Cortical Neuroplasticity and Cognitive Function in Early-Stage, Mild-Moderate Hearing Loss: Evidence of Neurocognitive Benefit From Hearing Aid Use. Frontiers in Neuroscience. 2020;14.

8. Anzivino R, Conti G, Di Nardo W, et al. Prospective evaluation of cognitive functions after rehabilitation with cochlear implant or hearing aids: Preliminary results of a multicentric study on elderly patients. American Journal of Audiology. 2019;28(3S):762-774.

9. Sarant J, Harris D, Busby P, et al. The Effect of Hearing Aid Use on Cognition in Older Adults: Can We Delay Decline or Even Improve Cognitive Function? Journal of Clinical Medicine. 2020;9(1):254.

10. Nguyen MF, Bonnefoy M, Adrait A, et al. Efficacy of hearing aids on the cognitive status of patients with Alzheimer's disease and hearing loss: a multicenter controlled randomized trial. Journal of Alzheimer's disease. 2017;58(1):123-137.

11. Gianopoulos I, Stephens D, Davis A. Follow up of people fitted with hearing aids after adult hearing screening: the need for support after fitting. BMJ. 2002;325(7362):471.

12. Hooper E, Simkin Z, Abrams H, et al. Feasibility of an intervention to support hearing and vision in dementia: the SENSE-Cog Field Trial. Journal of the American Geriatrics Society. 2019;67(7):1472-1477.

13. Deal JA, Goman AM, Albert MS, et al. Hearing treatment for reducing cognitive decline: Design and methods of the Aging and Cognitive Health Evaluation in Elders randomized controlled trial. Alzheimers Dement. 2018;4:499-507. 
14. Young A, Ferguson-Coleman E, Keady J. Understanding the personhood of Deaf people with dementia: Methodological issues. Journal of aging studies. 2014;31:62-69.

15. Wolski L, Leroi I, Regan J, et al. The need for improved cognitive, hearing and vision assessments for older people with cognitive impairment: a qualitative study. BMC Geriatrics. 2019;19(1):328.

16. Beck, DL, Flexer, C. Listening is where hearing meets brain... in children and adults. Hearing Review. 2011;18(2):30-35.

17. Beck DL, Danhauer JL. Amplification for adults with hearing difficulty speech in noise problems and normal thresholds. Journal of Otolaryngology-ENT Research. 2019;11(11).

18. Rowe C, Healy SD. Measuring variation in cognition. Behavioral ecology. 2014:25(6):1287-1292.

19. Boogert NJ, Madden JR, Morand-Ferron J, et al. Measuring and understanding individual differences in cognition. Philos Trans $R$ Soc Lond B Biol Sci. 2018;373(1756):20170280.

20. Clarke NA, Henshaw H, Akeroyd MA, Adams B, Hoare DJ. Associations between subjective tinnitus and cognitive performance: systematic review and meta-analyses. Trends in Hearing. 2020;in press.

21. Baguley D, Andersson G, McFerran D, et al. Tinnitus: A Multidisciplinary Approach. 2nd ed. Wiley-Blackwell; 2012.

22. Amieva H, Ouvrard C. Does Treating Hearing Loss in Older Adults Improve Cognitive Outcomes? A Review. Journal of Clinical Medicine. 2020;9(3):805

23. Livingston G, Sommerlad A, Orgeta V, Costafreda et al. Dementia prevention, intervention, and care. The Lancet. 2017;390(10113):2673-2734.

24. Dawes P, Emsley R, Cruickshanks KJ, et al. Hearing loss and cognition the role of hearing aids, social isolation and depression. PloS one. 2015;10(3):e0119616.

25. Stahl SM. Does treating hearing loss prevent or slow the progress of dementia? Hearing is not all in the ears, but who's listening? CNS spectrums. 2017;22(3):247-50.

26. Uchida Y, Sugiura S, Nishita Y, et al. Age-related hearing loss and cognitive decline - The potential mechanisms linking the two. Auris Nasus Larynx. 2019;46(1):1-9.

27. Rabbit PMA. Channel capacity, intelligibility and immediate memory. Quarterly Journal of Experimental Psychology. 1968;20(3):241-248.

28. Piquado T, Cousins KA, Wingfield A, et al. Effects of degraded sensory input on memory for speech: Behavioral data and a test of biologically constrained computational models. Brain research. 2010;1365:48-65.

29. Rabbit PMA. Mild hearing loss can cause apparent memory failure which increase with age and reduce with IQ. Acta Otolaryngoligica. 1991;476:167-176

30. Surprenant AM. Effects of noise on identification and serial recall of nonsense syllables in older and younger adults. Aging, Neuropsychology and Cognition. 2007;14(2):126-143.

31. Dryden A, Allen HA, Henshaw H, et al. The association between cognitive performance and speech-in-noise perception for adult listeners: A systematic literature review and meta-analysis. Trends in hearing 2017;21:2331216517744675.
32. Rogers CS, Peele JE. Interactions between audition and cognition in hearing loss and aging. In: The Auditory Cognitive Neuroscience of Speech Perception (Springer Handbook of Auditory Research) (Holt and Lott, eds). Springer.

33. Golub JS, Brickman AM, Ciarleglio AJ, et al. Association of subclinica hearing loss with cognitive performance. JAMA Otolaryngology-Head \& Neck Surgery. 2020;146(1):57-67.

34. Mamo SK, Reed NS, Sharrett AR, et al. Relationship Between DomainSpecific Cognitive Function and Speech-in-Noise Performance in Older Adults: The Atherosclerosis Risk in Communities Hearing Pilot Study. American Journal of Audiology. 2019;28(4):1006-14.

35. Gaeta L, Azzarello J, Baldwin J, et al. Effect of Reduced Audibility on Mini-Mental State Examination Scores. Journal of the American Academy of Audiology. 2019;30(10):845-55.

36. Deal JA, Betz J, Yaffe K, et al. Hearing impairment and incident dementia and cognitive decline in older adults: the Health ABC Study. Journals of Gerontology Series A: Biomedical Sciences and Medical Sciences. 2017;72(5):703-709.

37. Dawes P. 20Q: Hearing Loss and Dementia - Association, Link or Causation? Audiology Online. 2017.

38. Yuan J, Sun Y, Sang S, et al. The risk of cognitive impairment associated with hearing function in older adults: a pooled analysis of data from eleven studies. Scientific reports. 2018;8(1):1-10.

39. Jafari Z, Kolb BE, Mohajerani MH. Age-Related Hearing Loss and Tinnitus, Dementia Risk, and Auditory Amplification Outcomes. Ageing research reviews. 2019:100963.

40. Allen NH, Burns A, Newton V, et al. The effects of improving hearing in dementia. Age and Ageing. 2003;32(2):189-93.

41. Ray M, Dening T, Crosbie B. Dementia and hearing loss: A narrative review. Maturitas. 2019.

42. Panza F, Lozupone M, Sardone R, et al. Sensorial frailty: age-related hearing loss and the risk of cognitive impairment and dementia in later life. Therapeutic Advances in Chronic Disease. 2019;10:2040622318811000.

43. Deal JA, Albert MS, Arnold M, et al. A randomized feasibility pilot trial of hearing treatment for reducing cognitive decline: results from the Aging and Cognitive Health Evaluation in Elders Pilot Study. Alzheimer's \& Dementia: Translational Research \& Clinical Interventions. 2017;3(3):410-5.

44. Newman $\mathrm{AB}$, Bayles $\mathrm{CM}$, Milas $\mathrm{CN}$, et al. The 10 keys to healthy aging: findings from an innovative prevention program in the community. Journal of aging and health. 2010;22(5):547-566.

45. Jilla AM, Johnson CE, Danhauer JL, et al. Predictors of Hearing Aid Use in the Advanced Digital Era: An Investigation of Benefit, Satisfaction, and Self-Efficacy. J Am Acad Audiol. 2020;31(2):87-95.

46. Lawrence BJ, Jayakody DM, Henshaw H, et al. Auditory and cognitive training for cognition in adults with hearing loss: a systematic review and meta-analysis. Trends in hearing. 2018;22:2331216518792096.

47. Ferguson MA, Kitterick PT, Chong LY, et al. Hearing aids for mild to moderate hearing loss in adults. Cochrane Database of Systematic Reviews. 2017; 9 